\title{
Is it really nephroblastomatosis? Analysis of diagnostic and therapeutic difficulties: case report of three patients
}

\author{
Małgorzata Janeczko-Czarnecka', Ewa Niedzielska-Łoś', Krzysztof Bronowicki², \\ Wojciech Pietras' ${ }^{1}$, Marek Ussowicz' ${ }^{1}$

\begin{abstract}
'Department of Paediatric Bone Marrow Transplantation, Oncology, and Haematology, Wroclaw Medical University, Wroclaw, Poland

2Department of Paediatric Surgery, Marciniak Lower Silesian Specialist Hospital - Emergency Medicine Centre, Wroclaw Medical University, Wroclaw, Poland
\end{abstract}

\section{ABSTRACT}

Nephroblastomatosis (NB) is the presence of multifocal residues of embryonic kidney localised in the kidney capsule, the cortex, or in the renal core. A characteristic picture of lesions in imaging studies (ultrasound, computed tomography, magnetic resonance imaging) allows us to establish the diagnosis and start chemotherapy. We present a course of treatment in three patients with baseline diagnosis of NB. We emphasise that due to the lack of initial histopathological examination diagnosis of NB only by means of imaging studies can be both a diagnostic and a therapeutic challenge. Close cooperation of specialists in the fields of radiology, surgery, and paediatric oncology is crucial.

\section{KEY WORDS:}

nephrogenic rests, nephroblastomatosis, Wilms' tumour, SIOP 2001 protocol.

\section{INTRODUCTION}

Nephroblastomatosis (NB) is defined as the presence after the $36^{\text {th }}$ week of gestation of diffuse, multifocal nephrogenic rests (NR) located in the kidney capsule in the renal cortex or in the renal core [1]. Based on autopsy results, NR-like lesions are present in approximately $1 \%$ of newborns, and malignant transformation occurs in less than $1 \%$ of them [2]. NR were found in $30-44 \%$ patients with unilateral Wilms' tumour (WT) and in almost 99\% patients with synchronous or metachronous bilateral WT, and they are considered as pre-neoplastic lesions, a precursor for nephroblastoma $[3,4]$.

Beckwith et al. presented the classification of renal lesions, which were collectively described as NB, and de- fined NR as foci of improperly surviving nephrons containing cells that may in the future become the primary locus of WT $[5,6]$. Beckwith classified NR according to their location, distinguishing perilobar nephrogenic rests (PLNR), intralobar nephrogenic rests (ILNR), and mixed. The histopathological distinction can be relevant due to the different prognosis in each subtype of NB. PLNR are often multifocal and well bordered, while ILNR are monofocal and poorly demarcated from kidney parenchyma. Beckwith further distinguished sclerosing, dormant, hyperplastic, and neoplastic NR, with the highest risk of WT development within the hyperplastic and neoplastic NR [4]. In clinical practice we distinguish multifocal, microscopic NR related to WT and macroscopic lesions diagnosed incidentally, without the coexistence with WT. In the case of

\section{ADDRESS FOR CORRESPONDENCE:}

Małgorzata Janeczko-Czarnecka, Department of Paediatric Bone Marrow Transplantation, Oncology,

and Haematology, 213 Borowska St., 50-556 Wroclaw, Poland, ORCID: 0000-0001-6629-1435,

e-mail: ml.janeczko@gmail.com 


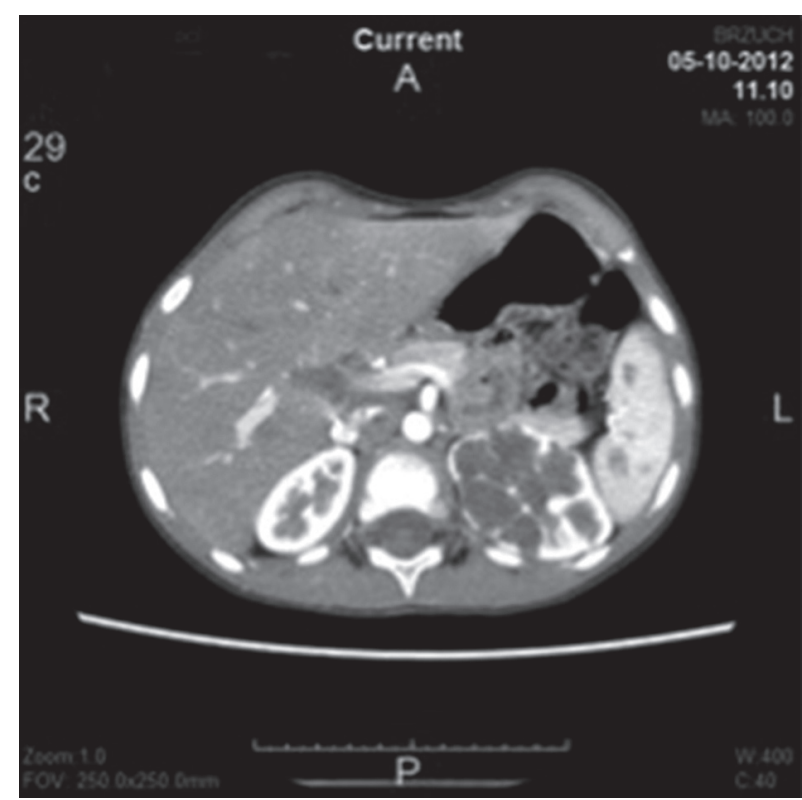

FIGURE 1. Abdominal CT scan at the diagnosis (patient 1). In the upper part of the left kidney a hypodense area of $4 \mathrm{~cm} \times 3.5 \mathrm{~cm} \times$ $7 \mathrm{~cm}$ in diameter, undergoing inhomogeneous strengthening and causing significant thinning of the kidney core. The lesion does not exceed the capsule of the kidney

microscopic lesions, the risk of metachronous WT is significantly increased. Preliminary diagnosis of NB as well as WT is based only on the results of imaging studies abdominal ultrasound (US), computed tomography (CT) or magnetic resonance imaging (MRI). The most characteristic feature of NB in the radiological studies is the image uniformity in all three methods both before and after administration of contrast agent. WT imaging usually reveals inhomogeneous lesions enhancing after the administration of contrast agent $[6,7]$. Radiological evaluation plays a very important role in the diagnostic process, monitoring the course of treatment and therapeutic decisions. In the case of unsatisfactory NB regression during chemotherapy or progression, surgery is recommended due to the high risk of developing WT. Because NB often occurs bilaterally, in most cases nephron sparing surgery (NSS) is preferred [8]. Biopsy is not recommended because it does not distinguish between hyperplastic NB and WT. According to the latest protocol SIOP 2001 after diagnosis based on the imaging studies, chemotherapy with vincristine at a dose of $1.5 \mathrm{mg} / \mathrm{m}^{2}$ and actinomycin D at a dose of $45 \mu \mathrm{g} / \mathrm{kg}$ should be started. The intensity and duration of treatment depends on the results of control imaging studies.

\section{CASE REPORTS}

We reviewed 56 WT patients hospitalised in years 2011-2014 in our Clinic and reported three patients with initial diagnosis of NB, treated according to SIOP 2001 protocol. None of these three patients had a family history of cancer or renal diseases, and no symptoms of WT1related disorders were observed.

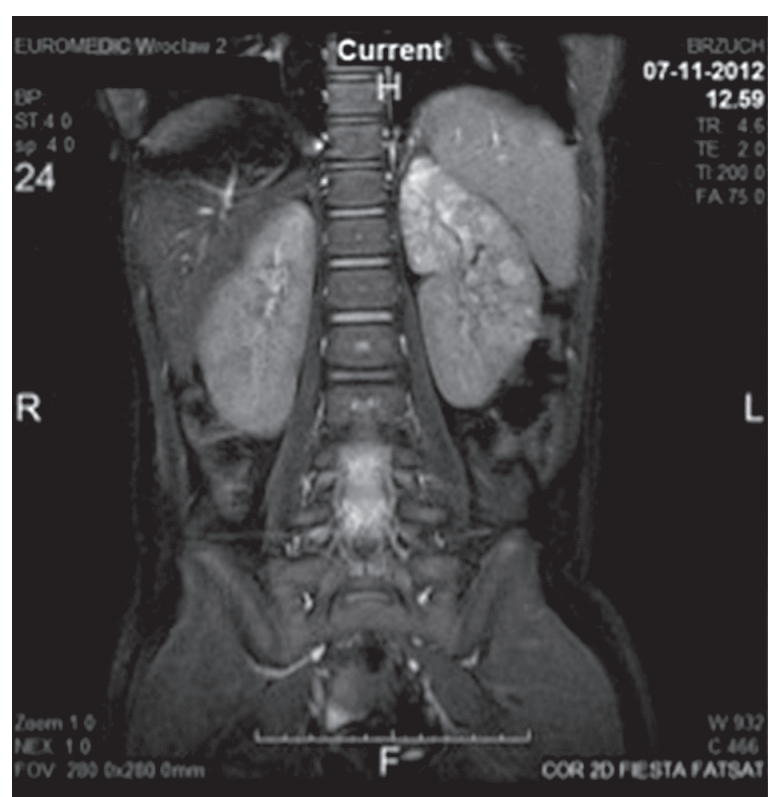

FIGURE 2. Abdominal CT scan after four weeks of chemotherapy (patient 1). No satisfactory regression of lesions

In a five-year-old girl (patient 1) both US and CT performed due to urine incontinence revealed a structure with heterogeneous solid and cystic areas with numerous microcalcifications in the upper part of the left kidney (Fig. 1). Chest X-ray was performed in order to rule out metastatic disease. On the basis of imaging studies a diagnosis of NB was established, and she started chemotherapy. After four weeks of treatment MRI did not show regression of lesions (Fig. 2), and NSS was performed. Histopathological examination showed polycystic kidney disease instead of NB. Currently the girl is in good general condition, without signs and symptoms of malignant disease.

An eight-year-old boy (patient 2) had abdominal CT revealing in the middle of the left kidney a nodular mass of size $31 \mathrm{~mm} \times 20 \mathrm{~mm} \times 17 \mathrm{~mm}$ sharply demarcated from the kidney parenchyma (Fig. 3). Chest CT did not reveal any metastatic lesions. Histopathological examination of the material obtained from the biopsy indicated NB. In the MRI after four weeks of chemotherapy the regression of the lesion was satisfactory, and the boy continued chemotherapy. After an additional four weeks of treatment both US and CT did not show further regression, and NSS was performed. Postoperative histological examination revealed stromal type WT. The boy underwent 27 weeks of postoperative chemotherapy for the standard-risk group. In the control MRI after treatment remission was confirmed and the patient remains alive and well.

A nine-month-old boy (patient 3) was diagnosed due to prenatally observed left kidney enlargement and asymmetry without signs of urinary retention. US re- 


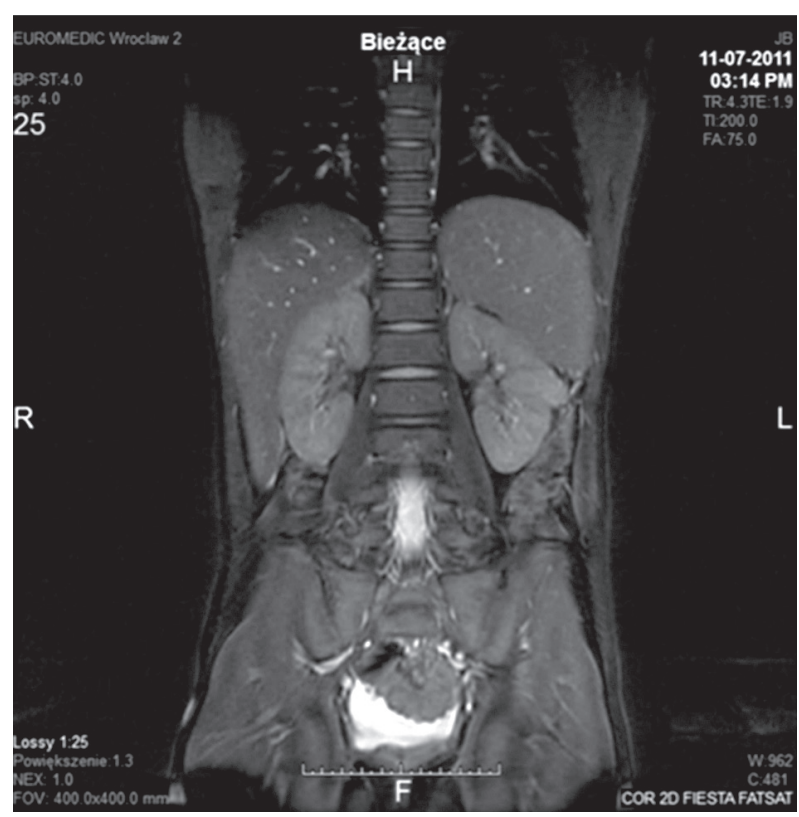

FIGURE 3. Abdominal CT scan at diagnosis (patient 2). Nodular mass in the middle of the left kidney, sharply demarked from the kidney core, $31 \mathrm{~mm} \times 20 \mathrm{~mm} \times 17 \mathrm{~mm}$ in diameter

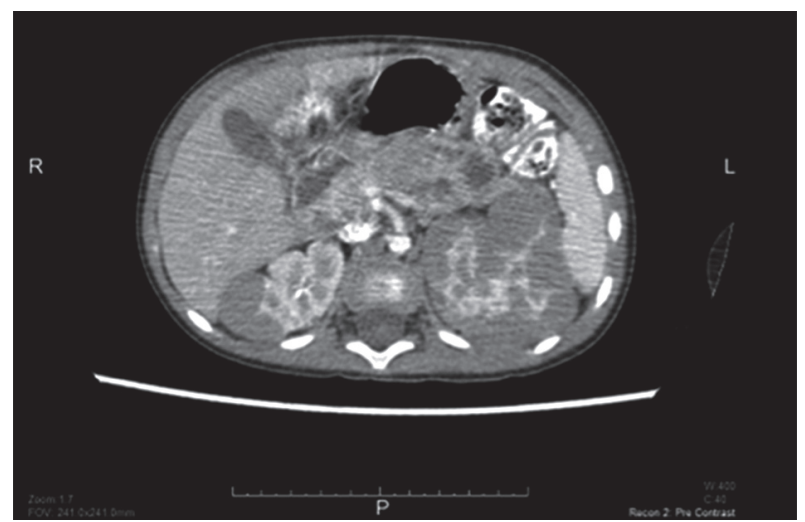

FIGURE 4. Abdominal CT scan at diagnosis (patient 3). Multiple nodular lesions in both kidneys. Enlargement of the left kidney

vealed numerous foci of mixed echostructure in both kidneys, while CT confirmed NB-like lesions in both kidneys (Fig. 4). Chest X-ray ruled out metastatic disease. Control CT made after seven weeks of treatment showed significant regression of lesions, and the boy continued the treatment according to the protocol until 12 months. MRI performed after completing the treatment confirmed complete regression of lesions, but after an additional 12 months MR revealed a vague lesion, arousing suspicion of WT. NSS was performed and a diagnosis of WT (intermediate-risk group, stage I) was established. The boy underwent 10 weeks of postoperative chemotherapy with vincristine. Currently, the boy is in good general condition and remains in remission.

\section{DISCUSSION}

Experience of our centre shows many diagnostic and subsequent therapeutic difficulties with NB. One of the first reports on the results of treatment of four patients (range: one month to two years) diagnosed with a massive NB was published by de Chadarévian et al. By using a combination of chemotherapy and irradiation, previously described foci of NB have completely regressed. These patients were not operated and remained in remission during the follow-up period from 10 to 44 months [9]. Haaddy et al. and Telander et al. reported cases of patients diagnosed with massive, disseminated, bilateral NB treated successfully with vincristine and actinomycin $\mathrm{D}[10,11]$. For paediatric patients in whom NB is usually diagnosed at a very young age, conservative treatment is a huge benefit. In these children complete remission can be achieved without the need for surgery, thus limiting the chance of irreversible damage to kidneys and the risk of renal failure, with dialysis and even kidney transplantation. Residual, non-regressing tumours must be verified due to possible misdiagnosis or the evolution to overt malignancy. Both our patients with NB (second and third) eventually proceeded to surgery and were diagnosed with progression to WT in solitary lesions, but preoperative therapy made the NSS eligible. This procedure enables the proper function of both kidneys to be maintained, and, considering the risk of developing additional lesions, it allows more radical treatment in the future, including nephrectomy, if necessary $[12,13]$.

Another issue is the relationship between bilateral metachronous NB and bilateral WT described by Copes et al. in 42 out of 58 patients diagnosed with WT [14]. The authors draw attention to the fact that children under the age of 12 months diagnosed with both NB and WT have significantly increased risk of developing the tumour in the contralateral kidney. Such patients require regular and long-term follow-up due to risk of delayed malignant transformation in affected kidneys. A similar clinical situation was observed in our youngest patient (third patient) initially diagnosed with bilateral NB. Bergeron et al. over a period of 11 years described the differences between a group of 18 patients with both WT and NB vs. 63 children with WT alone [3]. They observed a statistically shorter period of event-free survival and a significantly higher relapse incidence in the first group (50\% vs. $11 \%)$. In this group relapses occurred after a longer observation period ( 25 vs. 10 months). These findings justify the necessity of further and more frequent monitoring of patients with WT coexisting with NB, and extension of the observation period.

Perlman et al. described the diagnostic and therapeutic difficulties in 52 patients initially diagnosed with hyperplastic, perilobar NB [15]. Out of 33 patients who underwent diagnostic biopsy and adjuvant chemotherapy, in 18 patients WT developed after a median time of 35 months. In the second group, 16 children underwent nephrectomy combined with adjuvant chemotherapy, and only three patients were subsequently diagnosed with WT in the contralateral kidney within a mean fol- 
low-up period of 36 months. The third group consisted of three patients who, after biopsy confirming the diagnosis of NB, were left untreated, and all developed WT within a follow-up period of less than 10 months. The authors thus proved that hyperplastic, perilobar NB must be regarded as a pre-neoplastic lesion, and accurate diagnosis in cooperation with radiologists and paediatric surgeons combined with proper treatment enables long-term survival of these patients. Close follow-up of NB patients during and after chemotherapy and readiness to perform therapeutic intervention are emphasised in the literature due to lack of predictability of clinical course $[1,16,17]$.

\section{CONCLUSIONS}

Due to lack of initial histopathological examination, diagnosis of NB only by means of imaging studies can cause great diagnostic and therapeutic difficulties. Although the NB patients have a very good prognosis, the initiation and continuation of chemotherapy and decisions on surgical interventions require high levels of expertise and wariness due to the possibility of misdiagnosis or disease evolution. We also cannot forget that benign lesions of the kidney, like polycystic kidney disease, may occur, and we should also take them into consideration during differential diagnosis. Therefore, the cooperation of specialists in the fields of radiology, surgery, and oncology is crucial. NB is a very heterogeneous disease and can give rise to $\mathrm{WT}$ even years after therapy completion, so close monitoring of patients, alertness, and readiness to change the therapeutic strategy at each stage of treatment are very important.

\section{DISCLOSURE}

The authors declare no conflict of interest.

\section{REFERENCES}

1. Machmouchi M, Bayoumi M, Mamoun I, et al. Bilateral universal nephroblastomatosis in an 8-month-old infant treated with chemotherapy. Pediatr Nephrol 2005; 20: 1007-1010.

2. Prasil P, Laberge JM, Bond M, et al. Management decisions in children with nephroblastomatosis. Med Pediatr Oncol 2000; 35: 429-433.

3. Bergeron C, Iliescu C, Thiesse P, et al. Does nephroblastomatosis influence the natural history and relapse rate in Wilms' tumour? A single centre experience over 11 years. Eur J Cancer 2001; 37: 385-391.

4. Hennigar RA, O'Shea P, Grattan-Smith JD. Clinicopathologic features of nephrogenic rests and nephroblastomatosis. Adv Anat Pathol 2001; 8: 276-289.

5. Beckwith JB, Kiviat NB, Bonadio JF. Nephrogenic Rests, nephroblastomatosis, and the pathogenesis of Wilms' tumor. Pediatr Pathol 1990; 10: 1-36.

6. Lonergan GJ, Martínez-León MI, Agrons GA, et al. Nephrogenic Rests, Nephroblastomatosis, and Associated Lesions of the Kidney. Radiographics 1998; 18: 947-968.
7. Rohrschneider WK, Weirich A, Rieden K, Darge K, Troger J, Graf N. US, CT and MR imaging characteristics of nephroblastomatosis. Pediatr Radiol 1998, 28, 435-443.

8. Millar AJ, Davidson A, Rode H, et al. Nephron-sparing surgery for bilateral Wilms' tumours: a single-centre experience with 23 cases. Afr J Paediatr Surg 2011; 8: 49-56.

9. de Chadarévian JP, Fletcher BD, Chatten J, Rabinovitch HH. Massive infantile nephroblastomatosis: a clinical, radiological, and pathological analysis of four cases. Cancer 1977; 39: 2294-2305.

10. Haddy TB, Bailie MD, Bernstein J, et al. Bilateral, diffuse nephroblastomatosis: report of a case managed with chemotherapy. J Pediatr 1977; 90: 784-786.

11. Telander RL, Gilchrist GS, Burgert EO Jr, et al. Bilateral massive nephroblastomatosis in infancy. J Pediatr Surg 1978; 13: 163-166.

12. Rauth TP, Slone J, Crane G, et al. Laparoscopic nephron-sparing resection of synchronous Wilms tumors in a case of hyperplastic perilobar nephroblastomatosis. J Pediatr Surg 2011; 46: 983-988.

13. Cozzi DA, Zani A. Nephron-sparing surgery in children with primary renal tumor: indications and results. Semin Pediatr Surg 2006; 15: 3-9.

14. Coppes MJ, Arnold M, Beckwith JB, et al. Factors affecting the risk of contralateral Wilms tumor development: a report from the National Wilms Tumor Study Group. Cancer 1999; 85: 1616-1625.

15. Perlman EJ, Faria P, Soares A, et al. Hyperplastic Perilobar Nephroblastomatosis: Long-Term Survival of 52 Patients. Pediatr Blood Cancer 2006; 46: 203-221.

16. Sethi AT, Narla LD, Fitch SJ, Frable WJ. Wilms tumor in the setting of bilateral nephroblastomatosis. Radiographics 2010; 30: 14211425.

17. Stabouli S, Printza N, Dotis J, et al. Perilobar nephroblastomatosis: natural history and management. Case Rep Pediatr 2014; 2014: 756819. 